\title{
Análisis de la deserción estudiantil en una universidad pública de Bolivia
}

\section{Analysis of student dropout at a public university in Bolivia}

\author{
Juan Carlos Poveda Velasco ${ }^{1}$ (1) @ \\ Iván Marcelo Poveda Velasco' ${ }^{1}$ [D : \\ Irlanda América España Irala' 1 (1) @
}

${ }^{1}$ Universidad Mayor, Real y Pontificia de San Francisco Xavier de Chuquisaca (Bolivia).

\begin{abstract}
Resumen
La investigación tiene el objetivo de identificar los factores que influyen sobre la deserción universitaria de los estudiantes de la Universidad Mayor, Real y Pontificia de San Francisco Xavier de Chuquisaca; la investigación es de tipo aplicada, cuantitativa, descriptiva, cuasi-experimental. Busca entender mejor la deserción estudiantil a partir del análisis de ecuaciones estructurales, apoyado en el software SmartPLS versión 3. La población de referencia para el estudio es el total de estudiantes matriculados al inicio de la gestión 2017 (46.347 estudiantes), se realizó un muestreo probabilístico aleatorio para población finita conocida al $97 \%$ de confianza y $3 \%$ de error, con un total de 2.216 estudiantes encuestados (femenino 1.079, masculino 1.137). El análisis a través de ecuaciones estructurales determinó que los factores "Económico" y "Familiar" resultan ser los que tienen la mayor influencia en el abandono estudiantil, complementariamente se identificaron otros factores, como el de "Motivación", "Salud", "Social" y "Académico". Sobre la base de los resultados, se recomienda realizar investigaciones complementarias de cada factor identificado.
\end{abstract}

Palabras clave: Deserción universitaria; ecuaciones estructurales; educación superior.

\begin{abstract}
The research aims to identify the factors that influence the university dropout of the students of the Universidad Mayor, Real y Pontificia de San Francisco Xavier de Chuquisaca; the research is applied, quantitative, descriptive, quasi-experimental. It seeks to better understand student dropout from the analysis of structural equations, supported by SmartPLS version 3 software. The reference population for the study is the total number of students enrolled at the beginning of the 2017 management (46,347 students), a Random probabilistic sampling for the known finite population at $97 \%$ confidence and 3\% error, with a total of 2,216 students surveyed (female 1,079, male 1,137). The analysis through structural equations determined that the "Economic" and "Family" factors turn out to be those that have the greatest influence on student drop-out, in addition other factors were identified, such as "Motivation", "Health", "Social "and" Academic. "Based on the results, it's recommended to carry out complementary investigations of each identified factor.
\end{abstract} Keywords: University dropout; Structural equations; Higher education. 


\section{Introducción}

La deserción universitaria resulta ser un proceso bastante complejo que es abordado desde diferentes criterios, aunque es una temática estudiada desde hace varias décadas, sigue estando vigente en la problemática irresoluta de las instituciones de educación superior (Da Re \& Clerici, 2017; Escanés, Merlino, \& Ayllón, 2011; González-Ramírez, \& Pedraza-Navarro, 2017). Son diversos los factores socio-económicos estudiados para explicar las causas de la deserción en las universidades, con el fin de buscar políticas y estrategias institucionales que encuentren eliminar estas causas o por lo menos minimizar su efecto (González-Ramírez, \& Pedraza-Navarro, 2017; Piratoba, \& Barbosa, 2013; Rodriguez, \& Weise, 2006; Siles, \& Cortez, 2003).

El fenómeno de la deserción universitaria tiene múltiples efectos negativos, entre ellos se identifican los relacionados con la familia y los estudiantes, que ven afectadas las oportunidades de mejorar sus condiciones socio-laborales; el aumento de los costos para el financiamiento de las universidades por parte del Estado; el incremento en el gasto operacional y reducción de los índices académicos de las universidades, que tienen incidencia en los procesos de acreditación (Escanés et al., 2011; Medellín, 2010; Piratoba, \& Barbosa, 2013).

En Bolivia el Sistema de la Universidad Boliviana (SUB), está conformado por 15 universidades, 11 gratuitas y 4 privadas. El SUB en diez años ha tenido un crecimiento de aproximadamente el $72 \%$ en la matrícula estudiantil, pasando de 256.834 en el año 2004 a 440.918 en el año 2015. Las 11 universidades públicas gratuitas representan algo más del $75 \%$ de la matrícula universitaria total en Bolivia, registrando para el mismo periodo de tiempo una deserción definitiva promedio del $10.66 \%$ (CEUB, 2016). La generación de estadísticas sobre educación superior universitaria en Bolivia es limitada, de manera irregular se publican estadísticas sobre la matrícula, el crecimiento de la matrícula y sobre el presupuesto de manera agregada, existiendo muy poca o casi ninguna información sobre resultados de la gestión académica.

La Universidad Mayor, Real y Pontificia de San Francisco Xavier de Chuquisaca, forma parte del SUB, es pública y gratuita, siendo además la primera universidad de Bolivia y una de las más antiguas en América. La universidad tiene 15 facultades y más de 90 carreras en oferta; ha tenido un crecimiento vertiginoso en la matrícula universitaria, pasando de 26.480 estudiantes en el año 2004 a 46.347 estudiantes matriculados en el año 2017 (UMRPSFXCH/Dirección de Tecnologías de Información y Comunicación, 2017). Los últimos tres años la deserción universitaria tuvo un promedio del $18.01 \%$, un $8 \%$ por encima del promedio nacional, y con una 
tendencia aparente a aumentar (UMRPSFXCH/Dirección de Servicios Académicos, 2016); esto preocupa a las autoridades académicas universitarias, más aún cuando existe un programa de becas en efectivo que favorecen a estudiantes de familias con bajos recursos, que tengan un rendimiento académico aceptable.

Son varias las conjeturas que se realizan en torno a las causas que estarían provocando un nivel alto de deserción en relación al promedio nacional, por ello el problema investigativo propuesto es el siguiente: En el contexto de la universidad Mayor, Real y Pontificia de San Francisco Xavier de Chuquisaca, ¿cuáles son los factores que provocan la deserción universitaria?

En este sentido, el objetivo de la investigación es identificar los factores que influyen sobre la deserción universitaria de los estudiantes de la Universidad Mayor Real y Pontificia de San Francisco Xavier de Chuquisaca.

\subsection{Deserción universitaria}

La deserción universitaria es un concepto que se ha desarrollado progresivamente. En la actualidad, existe coincidencia en definirla como un abandono voluntario o inducido de la carrera elegida por el estudiante, tal como mencionan (Piratoba, \& Barbosa, 2013; Ramos, 2011; Sánchez, Navarro, \& García, 2009; Tinto, 1993) en sus investigaciones.

La deserción es el final de un proceso individual de conflicto, interno o externo, que termina en una auto-justificación de la decisión de abandonar los estudios universitarios, sin una adecuada valoración del efecto positivo o negativo del hecho mismo (Piratoba, \& Barbosa, 2013; Sánchez et al., 2009). Es una decisión personal, que puede ser el resultado de factores relacionados con las percepciones y sentimientos del estudiante, pero que también puede ser el resultado de factores determinados por el entorno socio económico en el que desarrolla sus actividades cotidianas, en la que de manera permanente o temporal hace que el estudiante abandone las aulas universitarias, independientemente del efecto que tenga en su vida futura (Ramos, 2011; Sánchez et al., 2009; Tinto, 1993).

\subsubsection{Factores para la deserción}

El abordaje de los factores que causan la deserción resulta ser diversos, tanto como los investigadores que han abordado la temática, para ello se han realizado abordajes desde el estudiante y sus motivaciones (Balmori, De la Garza, \& Guzmán, 2013; Escanés et al., 2011; Medellín, 2010); en relación a la interacción del 
estudiante con la universidad como institución en sus componentes de exigencia académica y aspectos organizacionales (Escanés et al., 2011; González-Ramírez, \& Pedraza-Navarro, 2017; Vries, León, Romero, \& Hernandez, 2011); además de como el entorno socio económico y ambiental influyen sobre el comportamiento del estudiante (Celis, Flores, Reyes, \& Venegas, 2013; Escanés et al., 2011; GonzálezRamírez, \& Pedraza-Navarro, 2017).

Hay varios modelos que proponen explicaciones sobre las causas que provocan la deserción universitaria, sin embargo, en la línea propuesta por (Balmori et al., 2013; Cabrera, Bethencourt, Alvarez, \& González, 2006; Escanés et al., 2011; GonzálezRamírez, \& Pedraza-Navarro, 2017), existe la necesidad de abordar el estudio de la problemática de la deserción universitaria desde un criterio multidimensional, que al mismo tiempo de incorporar las conceptualizaciones de los modelos resulte ser integrador de los mismos. Es menester hacer una revisión breve de los modelos de mayor aceptación. Los modelos más difundidos son:

- El modelo adaptativo, en él se presentan diferencias entre las estructuras culturales del estudiante con la cultura universitaria, provocando que el estudiante se sienta fuera de contexto y afectando su sentido de pertenencia (González-Ramírez, \& Pedraza-Navarro, 2017; Medellín, 2010; Sánchez et al., 2009).

- El modelo estructural, hace mención a las diferencias políticas, sociales y económicas de una universidad respecto a las del estudiante que pueden provocar la deserción (Cabrera et al., 2006; García de Fanelli, \& Adrogué de Deane, 2015), la institución universitaria resulta ser el reflejo de la cultura de un grupo social específico, que termina limitando las posibilidades de desarrollo de estudiantes con una procedencia diferente.

- El modelo economicista pretende hacer una relación costo / beneficio en el tiempo destinado a la permanencia en la universidad y las opciones laborales futuras (Cabrera et al., 2006; Celis et al., 2013; González-Ramírez, \& Pedraza-Navarro, 2017). Es decir, un estudiante permanecerá en una institución universitaria sólo si existe un retorno mayor al esfuerzo económico y temporal de su permanencia.

- El modelo psicológico hace referencia a un debilitamiento motivacional en los estudiantes en el transcurso del tiempo, provocando la deserción, (Cabrera et al., 2006; Escanés et al., 2011; Viale, 2014) al mencionar lo expuesto por Ajzen y Fishbein en 1975, quienes indican que progresivamente en algunos estudiantes se van debilitando sus aspiraciones iniciales a la universidad provocando su deserción. 
- El modelo pedagógico, en el que el rendimiento académico de los estudiantes (Cabrera et al., 2006; Viale, 2014) influye sobre sus aspiraciones futuras, el auto-concepto académico hace percibir las dificultades en los estudios, repercutiendo sobre las decisiones de deserción; adicionalmente la escasa formación previa al acceso a la universidad influye sobre variables relacionadas con el rendimiento académico, el auto-concepto académico e incluso la adaptación a la cultura institucional, provocando una disminución en la persistencia académica y la futura deserción.

- El modelo ecológico, habla sobre los contextos inmediatos y externos que influyen sobre el estudiante, entre los contextos inmediatos hace mención relevante al rol y presión familiar en diferentes momentos antes y durante la actividad universitaria del estudiante, que va desde el momento de elegir la carrera profesional como a la hora de valorar las calificaciones. Menciona también la relación entre la compatibilidad horaria para estudiar y asistir a clases, con la actividad laboral que desempeñen o con el tiempo destinado a la atención de sus propias familias. Factores relacionados con el acceso a una buena alimentación o atención en salud, también pueden repercutir sobre las decisiones de continuar los estudios (Cabrera et al., 2006; Da Re \& Clerici, 2017; Sánchez et al., 2009; Viale, 2014; Vries et al., 2011).

\subsection{Modelo estructural explicativo de la Deserción universitaria}

En los estudios empíricos realizados por (Balmori et al., 2013; Centro Microdatos, 2008; Escanés et al., 2011; Sánchez et al., 2009; Solano, Pérez, \& Uzcátegui, 2017), identifican como factores comunes que influyen sobre la deserción universitaria los aspectos motivacionales, académicos, económicos, sociales, familiares y de salud; a partir de estos estudios previos en los que se aplicaron encuestas y los modelos resumidos en el acápite 1.1.1. Factores para la deserción, se proponen las hipótesis explicativas del modelo estructural para el problema planteado.

Problema: ¿Cuáles son los factores que provocan la deserción universitaria?

H1: Lo Económico es un factor que influye sobre la deserción universitaria H2: La Salud es un factor que influye sobre la deserción universitaria H3: Lo Académico es un factor que influye sobre la deserción universitaria H4: Lo Motivacional es un factor que influye sobre la deserción universitaria H5: Lo Social es un factor que influye sobre la deserción universitaria H6: Lo Familiar es un factor que influye sobre la deserción universitaria 
El siguiente gráfico muestra el modelo propuesto para la investigación:

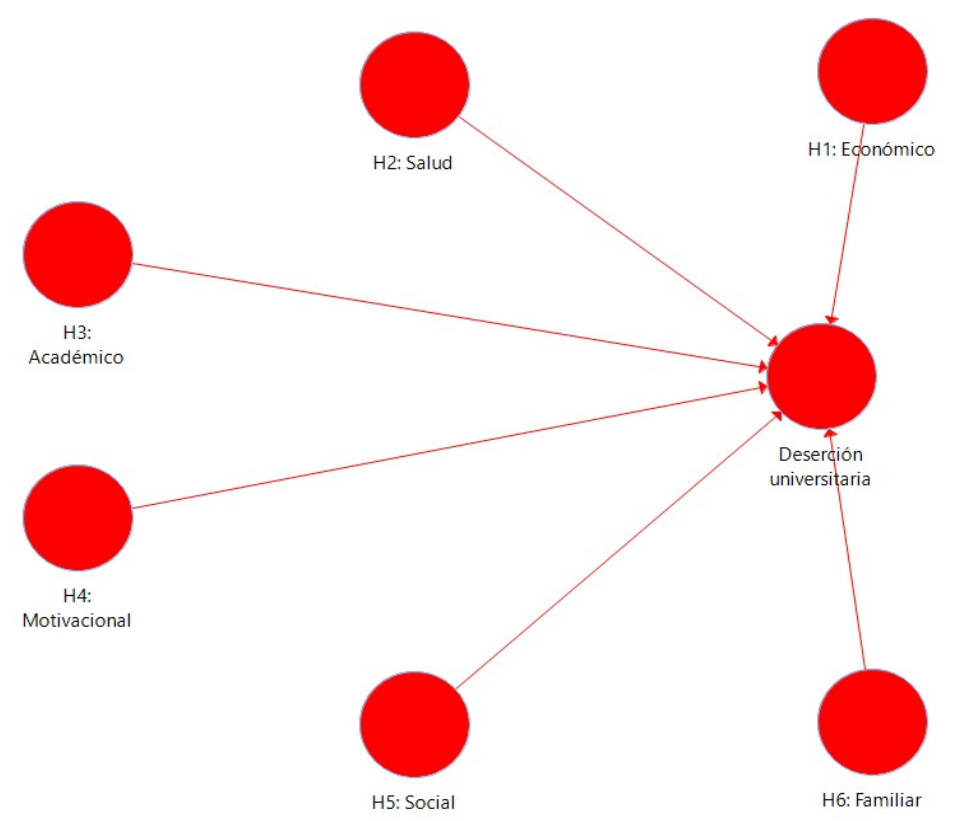

156 Figura 1. Modelo estructural propuesto.

Fuente: Elaboración propia del modelo. Gráfico del SmartPLS V.3

\section{Método}

La investigación es de tipo aplicada, cuantitativa, descriptiva, cuasi-experimental. Aplicada, porque contribuye a entender mejor el problema de la deserción estudiantil en la universidad San Francisco Xavier de Chuquisaca; es cuantitativa al medir los factores que influyen en la deserción con el apoyo de ecuaciones estructurales como método de análisis; es descriptiva al caracterizar la deserción a partir de los factores que la provocan; es cuasi-experimental al estudiar la relación causa / efecto entre la deserción y los factores que la generan. Asimismo, se aplica el método hipotético-deductivo y de investigación transversal (Morles, 2002; Morone, 2015; Robles, 2018; Ruiz, 2007; Sabino, 1992).

La población de referencia para el estudio la constituye el total de estudiantes matriculados en la gestión 2017, siendo de 46.347 estudiantes. El proceso de preparación para la recolección de datos fue realizado en diferentes fases. 
a) Primera fase, se elaboró un cuestionario primario organizado según el modelo estructural propuesto, a partir de la revisión de estudios previos realizados por (Balmori et al., 2013; Centro Microdatos, 2008; Escanés et al., 2011; Sánchez et al., 2009; Solano et al., 2017); se utiliza el total de preguntas de los estudios revisados, adecuando la redacción al lenguaje local y evitando que una pregunta se repita en su forma o contenido.

b) Segunda fase, para la validación de las preguntas, se aplica un cuestionario borrador a un grupo de sesenta (60) estudiantes elegidos aleatoriamente, tomando cuatro (4) estudiantes por cada facultad (2 femenino, 2 masculino). Se aplica una valoración a través de una escala Likert, con tres alternativas ("de acuerdo", "no corresponde", "en desacuerdo"). Las preguntas del cuestionario borrador que fueron marcadas por el total de estudiantes con la opción "no corresponde", son excluidas del cuestionario final.

c) Tercera fase, se reunió en un taller a sesenta (60) estudiantes, cuatro (4) por facultad, elegidos por los centros de estudiantes facultativos, con la condición que no hubieran participado en el evento anterior, de los cuales 2 debían ser dirigentes y 2 estudiantes de base; además, debería guardarse criterios de equidad de género. En el taller se conformaron cinco grupos tomando en cuenta las cinco grandes áreas del conocimiento que gestiona la Universidad de San Francisco Xavier de Chuquisaca, estas son: Ciencias Agrarias y Forestales, Ciencias de la Salud, Ciencias Económicas, Ciencias Sociales y Humanistas, Ciencias Tecnológicas. A estos grupos se realizó una breve explicación del objetivo de la investigación, así como una conceptualización básica de la deserción universitaria. A continuación se les solicitó que identificarán cuales serían las causas probables para la deserción universitaria, para ello se siguió el siguiente procedimiento:

- Primero, cada persona de manera individual debía elegir mediante una afirmación tres causas probables para la deserción.

- Segundo, cada persona mediante tarjetas escritas pone en un tablero las afirmaciones con causas probables para la deserción.

- Tercero, se agrupan las tarjetas según semejanza de las causas identificadas. No se excluye ninguna.

- Cuarto, se redacta una afirmación que refleje la idea de cada grupo de tarjetas semejantes.

- Quinto, se elabora en un papelógrafo el listado de las afirmaciones de causas probables identificadas por todos los miembros del grupo.

- Sexto, en una plenaria se presentan los cinco papelógrafos.

- Séptimo, se elabora un solo listado con todas las causas probables presentadas por los grupos. 
d) Cuarta fase, se realiza el análisis comparado del cuestionario final obtenido en la segunda fase con el listado de afirmaciones de la tercera fase, de manera que aquellas preguntas que tenían coincidencia con las causas probables identificadas en la Tercera fase, formen parte del cuestionario final a ser aplicado para la recolección de datos de la investigación. Como resultado se obtuvo un cuestionario con preguntas cerradas de alternativas de respuestas delimitadas, de tipo dicotómicas, de selección múltiple y escala de Likert con cinco opciones ("totalmente en Desacuerdo", "en Desacuerdo", "No corresponde", "de Acuerdo", "totalmente de Acuerdo").

e) Quinta fase, el cuestionario fue aplicado en una encuesta piloto a 90 estudiantes (45 femenino, 45 masculino), 6 por cada facultad, que fueron elegidos de manera aleatoria en coordinación con la Federación Universitaria Local-asociación que representa a todos los estudiantes de la universidad-. A los datos obtenidos mediante la encuesta piloto, se aplicó el análisis de bootstrapping para verificar la relación entre las cargas estadísticas de los indicadores y si las relaciones entre variables son significativas. Para ello se considera que el valor t-Statistic de las cargas y las relaciones entre variables sea mayor a 1,96 (Ledesma, 2008; Mendez-Gimenez, Fernández-Río, \& Cecchini-Estrada, 2014; Miranda, 2003). El análisis fue realizado con apoyo del software SmartPLS v.3. Concluido el análisis a 5000 interacciones, se identifica que la variable que corresponde a la pregunta bajo el código "Fa06", es la única que se encuentra por debajo del valor 1,96, por lo que se toma la decisión de excluirla del cuestionario.

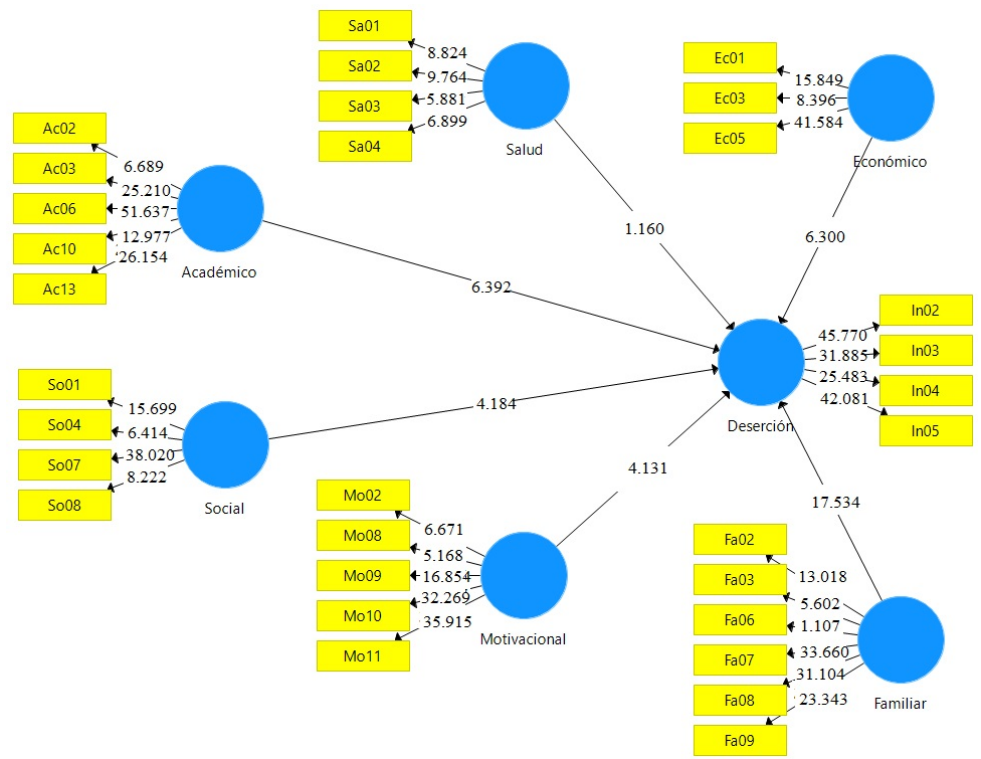

Figura 2. Resultado de análisis bootstrapping

Fuente: Datos de la aplicación de la Encuesta Piloto. Gráfico del SmartPLS V.3 
Realizado el ajuste, se hace correr una vez más el análisis bootstrapping, para verificar que todas las variables tengan valores por encima de 1,96; concluido el análisis se corroboró que los valores de las variables están por encima del valor requerido, con lo que el cuestionario quedo definido para su aplicación.

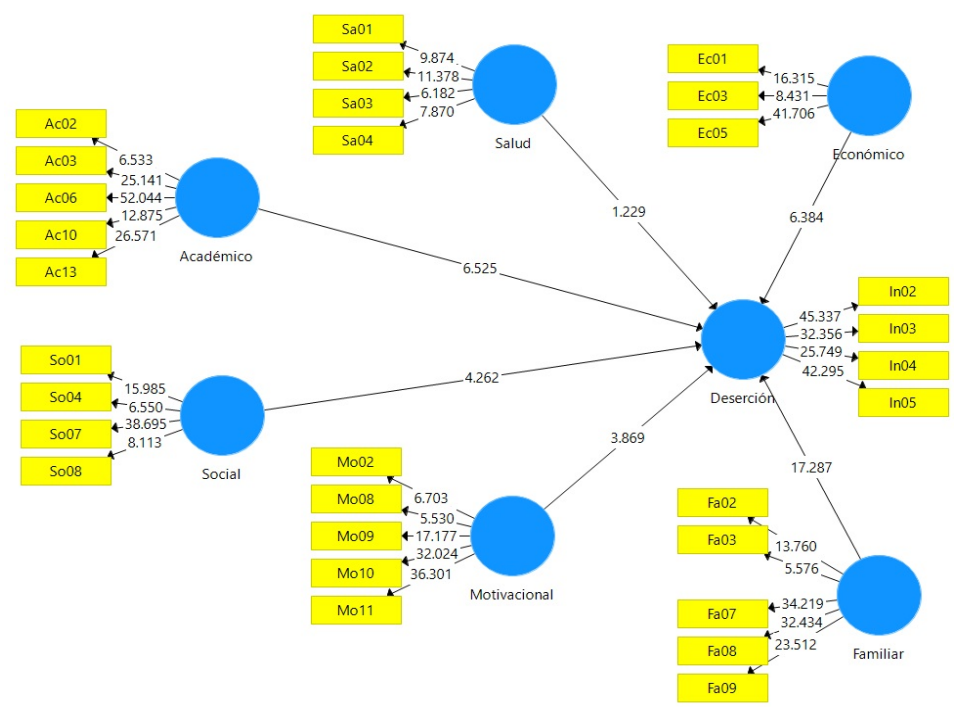

Figura 3. Análisis bootstrapping final con la exclusión de variable Fa06

Fuente: Datos de la aplicación de la Encuesta Piloto. Gráfico del SmartPLS V.3

En la gestión 2017, la población de estudiantes con matrícula regular de la Universidad de San Francisco Xavier de Chuquisaca fue de 46.347; para la aplicación de la encuesta (ver Anexo 1), se realizó un muestreo probabilístico aleatorio para población finita conocida, al 97\% de confianza y 3\% de error, dando como resultado una muestra de 2.216 (femenino 1.079, masculino 1.137), cuestionarios a ser aplicados. El cuestionario se realizó de manera virtual a través de la plataforma del sistema de información académica para estudiantes de la Universidad Mayor, Real y Pontificia de San Francisco Xavier de Chuquisaca.

\section{Resultados, análisis y discusión}

\subsection{Validez y confiabilidad del modelo}

Para la presentación y discusión de resultados, primero se recurrió a la valoración de fiabilidad del modelo propuesto, a través del análisis factorial confirmatorio basado en el criterio de Fornell y Larcker; Posteriormente se aplicó el análisis de validez convergente discriminante para los ítems de los factores que explican 
la deserción universitaria en San Francisco Xavier de Chuquisaca (Calvo-Porral, Martínez-Fernández, \& Juanatey-Boga, 2013; Leyva, \& Olague, 2014; RamirezCorrea \& Mariano, 2014).

Según el criterio de Fornell y Larcker propuesto en 1981 (Leyva, \& Olague, 2014; Martínez, \& Martínez, 2009), el análisis factorial confirmatorio del modelo es analizado a partir de la existencia de una validez discriminante entre dos variables latentes, si la varianza compartida entre pares de constructos es menor que la varianza extraída para cada constructo individual, en ese sentido en la tabla 1 se verifica que la varianza de cada factor es mayor a las compartidas, mostrando que el modelo de manera general resulta ser fiable.

Tabla 1. Evaluación inicial del modelo. Criterio Fornell y Larcker

\begin{tabular}{|c|c|c|c|c|c|c|c|}
\hline Factor & $\begin{array}{c}\text { Deserción } \\
\text { universitaria }\end{array}$ & $\begin{array}{c}\text { H1: } \\
\text { Económico }\end{array}$ & $\begin{array}{l}\text { H2: } \\
\text { Salud }\end{array}$ & $\begin{array}{c}\text { H3: } \\
\text { Académico }\end{array}$ & $\begin{array}{c}\text { H4: } \\
\text { Motivacional }\end{array}$ & $\begin{array}{l}\text { H5: } \\
\text { Social }\end{array}$ & $\begin{array}{l}\text { H6: } \\
\text { Familiar }\end{array}$ \\
\hline Deserción universitaria & 0.693 & & & & & & \\
\hline H1: Económico & 0.341 & 0.678 & & & & & \\
\hline H2: Salud & -0.263 & -0.283 & 0.597 & & & & \\
\hline H3: Académico & 0.368 & 0.222 & -0.226 & 0.630 & & & \\
\hline H4: Motivacional & 0.425 & 0.163 & -0.274 & 0.306 & 0.577 & & \\
\hline H5: Social & 0.403 & 0.248 & -0.297 & 0.292 & 0.512 & 0.586 & \\
\hline H6: Familiar & 0.587 & 0.303 & -0.283 & 0.312 & 0.477 & 0.404 & 0.563 \\
\hline
\end{tabular}

Fuente: Encuesta propia. Tabla elaborada por el SmartPLS V.3

Adicionalmente se realiza la valoración de cargas cruzadas (Leyva Cordero, \& Olague, 2014; Ramirez-Correa, \& Mariano, 2014), en la que se evidencia que los diferentes ítems tienen una carga superior comparada con los otros ítems, reflejando que el modelo es fiable, sin embargo, la carga cruzada de varios ítems es menor a 0.5 según el mínimo aceptable por el criterio de Fornell y Larcker (Calvo-Porral, Martínez-Fernández, et al., 2013; Ramirez-Correa, \& Mariano, 2014).

Tabla 2. Evaluación de cargas cruzadas. Criterio Fornell y Larcker

\begin{tabular}{|c|c|c|c|c|c|c|c|c|}
\hline Factor & Ítem & $\begin{array}{c}\text { Deserción } \\
\text { universitaria }\end{array}$ & $\begin{array}{c}\text { H1: } \\
\text { Económico }\end{array}$ & $\begin{array}{l}\text { H2: } \\
\text { Salud }\end{array}$ & $\begin{array}{c}\text { H3: } \\
\text { Académico }\end{array}$ & $\begin{array}{c}\mathrm{H} 4: \\
\text { Motivacional }\end{array}$ & $\begin{array}{l}\text { H5: } \\
\text { Social }\end{array}$ & $\begin{array}{c}\text { H6: } \\
\text { Familiar }\end{array}$ \\
\hline \multirow{5}{*}{ 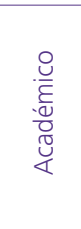 } & $\mathrm{AcO} 2$ & $-0,071$ & $-0,015$ & 0,178 & $-0,320$ & $-0,173$ & $-0,153$ & $-0,042$ \\
\hline & $\mathrm{Ac03}$ & 0,274 & 0,136 & $-0,118$ & 0,686 & 0,207 & 0,205 & 0,231 \\
\hline & $\mathrm{Ac06}$ & 0,338 & 0,129 & $-0,156$ & 0,803 & 0,249 & 0,217 & 0,256 \\
\hline & Ac10 & 0,145 & 0,243 & $-0,160$ & 0,531 & 0,116 & 0,147 & 0,141 \\
\hline & Ac13 & 0,210 & 0,188 & $-0,182$ & 0,695 & 0,220 & 0,210 & 0,220 \\
\hline
\end{tabular}




\begin{tabular}{|c|c|c|c|c|c|c|c|c|}
\hline Factor & Ítem & $\begin{array}{c}\text { Deserción } \\
\text { universitaria }\end{array}$ & $\begin{array}{c}\text { H1: } \\
\text { Económico }\end{array}$ & $\begin{array}{l}\text { H2: } \\
\text { Salud }\end{array}$ & $\begin{array}{c}\text { H3: } \\
\text { Académico }\end{array}$ & $\begin{array}{c}\text { H4: } \\
\text { Motivacional }\end{array}$ & $\begin{array}{l}\text { H5: } \\
\text { Social }\end{array}$ & $\begin{array}{c}\text { H6: } \\
\text { Familiar }\end{array}$ \\
\hline \multirow{4}{*}{ 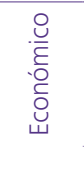 } & Ec01 & $-0,158$ & $-0,642$ & 0,276 & $-0,090$ & $-0,019$ & $-0,139$ & $-0,143$ \\
\hline & $\mathrm{ECO3}$ & 0,182 & 0,455 & $-0,086$ & 0,197 & 0,169 & 0,159 & 0,187 \\
\hline & Ec05 & 0,312 & 0,872 & $-0,224$ & 0,164 & 0,131 & 0,200 & 0,262 \\
\hline & $\mathrm{FaO2}$ & 0,270 & 0,037 & $-0,034$ & 0,100 & 0,211 & 0,173 & 0,466 \\
\hline \multirow{4}{*}{ 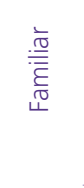 } & $\mathrm{FaO3}$ & 0,155 & 0,108 & $-0,148$ & 0,122 & 0,019 & 0,035 & 0,246 \\
\hline & $\mathrm{Fa} 07$ & 0,382 & 0,309 & $-0,271$ & 0,268 & 0,277 & 0,282 & 0,682 \\
\hline & $\mathrm{Fa08}$ & 0,396 & 0,166 & $-0,173$ & 0,205 & 0,485 & 0,395 & 0,686 \\
\hline & Fa09 & 0,384 & 0,187 & $-0,156$ & 0,157 & 0,225 & 0,155 & 0,611 \\
\hline \multirow{4}{*}{ 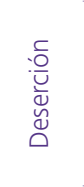 } & $\ln 02$ & 0,751 & 0,389 & $-0,258$ & 0,240 & 0,231 & 0,278 & 0,429 \\
\hline & $\ln 03$ & 0,674 & 0,134 & $-0,167$ & 0,226 & 0,294 & 0,264 & 0,384 \\
\hline & $\ln 04$ & 0,616 & 0,106 & $-0,119$ & 0,379 & 0,328 & 0,261 & 0,359 \\
\hline & $\ln 05$ & 0,725 & 0,285 & $-0,175$ & 0,189 & 0,333 & 0,311 & 0,450 \\
\hline \multirow{5}{*}{ 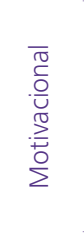 } & Mo02 & $-0,122$ & $-0,017$ & 0,143 & $-0,116$ & $-0,314$ & $-0,145$ & $-0,107$ \\
\hline & Mo08 & $-0,095$ & $-0,006$ & 0,158 & $-0,102$ & $-0,275$ & $-0,123$ & $-0,040$ \\
\hline & Mo09 & 0,235 & 0,143 & $-0,243$ & 0,174 & 0,550 & 0,265 & 0,316 \\
\hline & Mo10 & 0,325 & 0,126 & $-0,128$ & 0,233 & 0,762 & 0,395 & 0,344 \\
\hline & Mo11 & 0,339 & 0,112 & $-0,184$ & 0,224 & 0,778 & 0,415 & 0,393 \\
\hline \multirow{4}{*}{$\frac{\bar{D}}{\stackrel{D}{n}}$} & $\mathrm{Sa} 01$ & $-0,136$ & $-0,284$ & 0,637 & $-0,151$ & $-0,115$ & $-0,166$ & $-0,119$ \\
\hline & $\mathrm{SaO2}$ & $-0,172$ & $-0,203$ & 0,688 & $-0,155$ & $-0,150$ & $-0,202$ & $-0,168$ \\
\hline & $\mathrm{SaO3}$ & $-0,073$ & $-0,123$ & 0,425 & $-0,110$ & $-0,115$ & $-0,151$ & $-0,068$ \\
\hline & $\mathrm{SaO} 4$ & 0,202 & 0,094 & $-0,603$ & 0,127 & 0,239 & 0,188 & 0,254 \\
\hline \multirow{4}{*}{$\begin{array}{l}\bar{\pi} \\
\bar{\complement} \\
\backsim\end{array}$} & So01 & 0,247 & 0,266 & $-0,179$ & 0,224 & 0,214 & 0,618 & 0,247 \\
\hline & So04 & $-0,112$ & 0,021 & 0,117 & $-0,073$ & $-0,167$ & $-0,380$ & $-0,074$ \\
\hline & So07 & 0,355 & 0,190 & $-0,239$ & 0,247 & 0,502 & 0,819 & 0,371 \\
\hline & So08 & $-0,141$ & $-0,030$ & 0,139 & $-0,056$ & $-0,206$ & $-0,418$ & $-0,134$ \\
\hline
\end{tabular}

Fuente: Encuesta propia. Tabla elaborada por el SmartPLS V.3

La fiabilidad del modelo propuesto, confirma que según la validez discriminante de varianza comparada, los factores propuestos (H1: Económico, H2: Salud, H3: Académico, H4: Motivacional, H5: Social, H6: Familiar) explican de manera general la deserción universitaria, pero al realizar el análisis de cargas cruzadas se verifica que existen ítems que se encuentran con valores por debajo del mínimo aceptable, es por ello que debe realizarse el análisis individual de los factores según los ítems que los explican.

La segunda etapa analiza la influencia de cada factor sobre la deserción a partir de los ítems que influyen sobre cada factor, al tratarse de un estudio de carácter social se recurre a la evaluación de confiabilidad del modelo a partir de la validez interna, la fiabilidad compuesta y la varianza extraída media; se parte del análisis 
inicial realizado por el SmartPLS V3, donde se muestran los valores de confiabilidad, expresados por la correlación simple, donde los indicadores con índices menores a 0.7 deben ser excluidos por considerarse poco confiables. (Calvo-Porral, MartínezFernández, et al., 2013; Leyva, \& Olague, 2014; Ramirez-Correa, \& Mariano, 2014).

La Figura 4 muestra el resultado del análisis de confiabilidad inicial realizado por el SmartPLS V.3; a partir de este primer análisis se realiza la depuración progresiva de los valores menores a 0.7

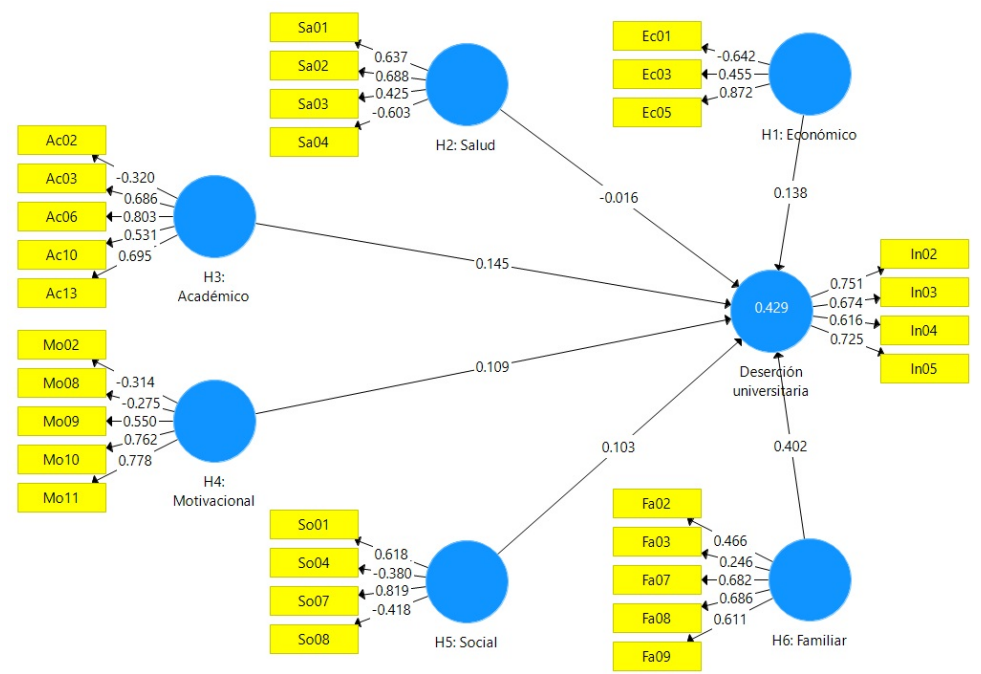

Figura 4. Fiabilidad interna individual del modelo. Análisis inicial.

Fuente: Encuesta sobre deserción universitaria 2017. Gráfico del SmartPLS V.3

La depuración de valores se realiza de manera progresiva y uno a la vez, hasta que todas las preguntas tengan un valor igual o mayor a 0.7 . Por ejemplo, en la figura 4. Fiabilidad interna individual del modelo. Análisis inicial. en el factor "H6: Familiar", la pregunta que corresponde a la codificación "Fa03" tiene un valor de 0.246, menor a 0.7 , al mismo tiempo que esta pregunta tiene el menor valor absoluto en relación a las otras preguntas; entonces se elimina esta pregunta y se vuelve a correr el análisis de fiabilidad. A continuación se verifica nuevamente los valores que corresponden a las preguntas, y si existe alguno que sea menor a 0.7 se procede a eliminarlo del modelo y hacer correr el análisis. Esta operación se realiza tantas veces sea necesario, hasta que todos los valores sean iguales o mayores a 0.7. 
Realizada la depuración progresiva de los valores se obtiene un nuevo gráfico representativo de la fiabilidad del modelo, la figura 5 . Fiabilidad individual interna del modelo. Análisis final. muestra el resultado final de las sucesivas depuraciones, donde se observa que todos los valores que están relacionados con las preguntas son mayores a 0.7 .

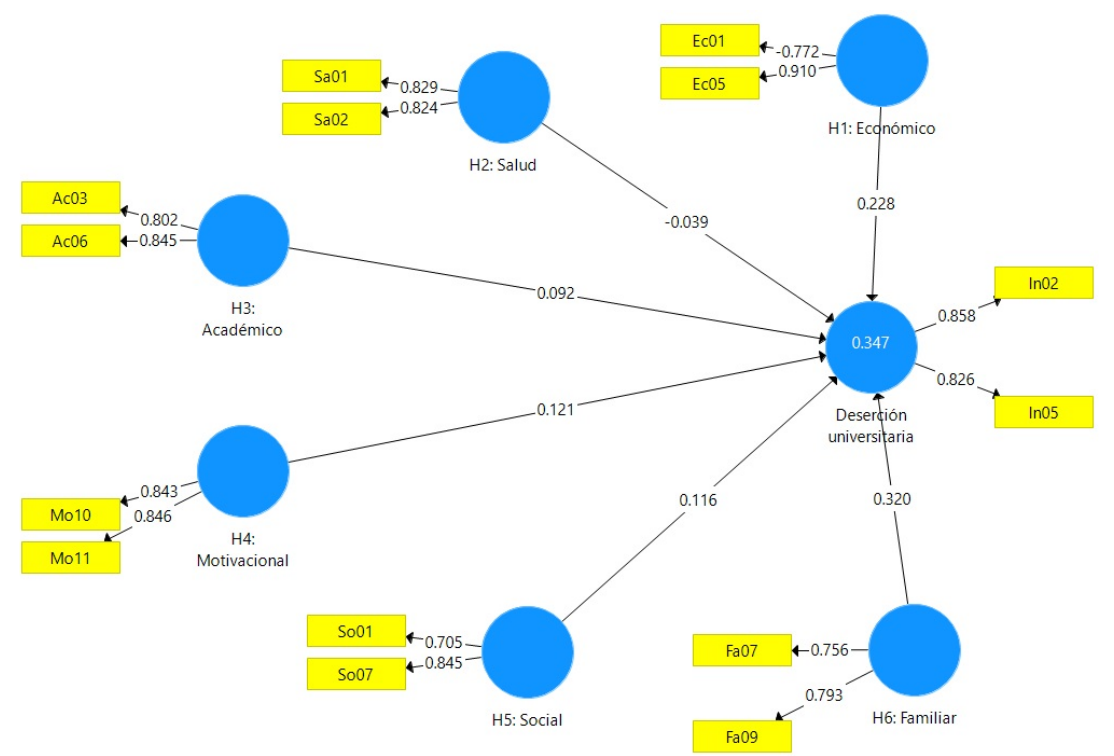

Figura 5. Fiabilidad individual interna del modelo. Análisis final.

Fuente: Encuesta sobre deserción universitaria 2017. Gráfico del SmartPLS V.3

Una vez verificada la fiabilidad individual interna del modelo se procede a la evaluación de confiabilidad a partir del Alfa de Cronbach, Fiabilidad compuesta y la Varianza extraída media.

El test con el coeficiente de fiabilidad de constructo muestra que los indicadores agrupados se correlacionan conjuntamente con sus variables, recomendando que el valor para el alfa de Cronbach y la Fiabilidad compuesta debe ser igual o mayor a 0.7; en relación al test de validez convergente, que permite identificar las consistencias internas del modelo, se recomienda que la Varianza Extraída Media (AVE) debe tener un valor mínimo de 0.5 , significando que más del $50 \%$ de la varianza del constructo se encuentra explicada por sus propios indicadores, estando altamente correlacionados (Calvo-Porral, Martinez-Fernández, Juanatey-Boga, \& Lévy-Mangín, 2013; Ramirez-Correa \& Mariano, 2014). 
La tabla 3 muestra los valores obtenidos para los indicadores de fiabilidad y validez del modelo corregido, en el que se puede verificar que todos los factores tienen valores mayores a los parámetros requeridos para cada uno de los indicadores.

Tabla 3. Fiabilidad y validez del modelo.

\begin{tabular}{lccc}
\hline \multicolumn{1}{c}{ Factor } & $\begin{array}{c}\text { Alfa de Cronbach } \\
\geq 0.7\end{array}$ & $\begin{array}{c}\text { Fiabilidad compuesta } \\
\geq 0.7\end{array}$ & $\begin{array}{c}\text { Varianza extraída } \\
\text { media (AVE) } \\
\geq 0.5\end{array}$ \\
\hline Deserción universitaria & 0,890 & 0,830 & 0,709 \\
H1: Económico & 0,872 & 0,732 & 0,713 \\
H2: Salud & 0,836 & 0,812 & 0,683 \\
H3: Académico & 0,827 & 0,808 & 0,678 \\
H4: Motivacional & 0,897 & 0,832 & 0,713 \\
H5: Social & 0,755 & 0,753 & 0,605 \\
H6: Familiar & 0,734 & 0,750 & 0,600 \\
\hline
\end{tabular}

Fuente: Encuesta propia. Tabla elaborada por el SmartPLS V.3

Finalmente, se realiza el test de validez discriminante con el fin de conocer el grado de diferencia entre las variables latentes del modelo, un modelo posee validez discriminante cuando la raíz cuadrada de la varianza media extraída AVE de cada variable latente es mayor a las correlaciones de Pearson del resto las variables latentes del modelo (Leyva Cordero, \& Olague, 2014; Ramirez-Correa, \& Mariano, 2014). La tabla 4 muestra los resultados obtenidos, donde los valores en negrilla son la raíz cuadrada de las AVE de cada variable y son mayores que las que se encuentran debajo en la misma columna, con lo que se cumple la condición.

Tabla 4. Validez discriminante del modelo corregido

\begin{tabular}{|c|c|c|c|c|c|c|c|}
\hline Factor & $\begin{array}{c}\text { Deserción } \\
\text { universitaria }\end{array}$ & $\begin{array}{c}\text { H1: } \\
\text { Económico }\end{array}$ & $\begin{array}{l}\mathrm{H} 2 \text { : } \\
\text { Salud }\end{array}$ & $\begin{array}{c}\text { H3: } \\
\text { Académico }\end{array}$ & $\begin{array}{c}\text { H4: } \\
\text { Motivacional }\end{array}$ & $\begin{array}{l}\text { H5: } \\
\text { Social }\end{array}$ & $\begin{array}{c}\text { H6: } \\
\text { Familiar }\end{array}$ \\
\hline Deserción universitaria & 0,842 & & & & & & \\
\hline H1: Económico & 0,374 & 0,844 & & & & & \\
\hline H2: Salud & $-0,212$ & $-0,308$ & 0,826 & & & & \\
\hline H3: Académico & 0,256 & 0,102 & $-0,128$ & 0,824 & & & \\
\hline H4: Motivacional & 0,306 & 0,099 & $-0,090$ & 0,253 & 0,844 & & \\
\hline H5: Social & 0,351 & 0,239 & $-0,182$ & 0,258 & 0,460 & 0,778 & \\
\hline H6: Familiar & 0,475 & 0,266 & $-0,185$ & 0,238 & 0,258 & 0,295 & 0,775 \\
\hline
\end{tabular}

Fuente: Encuesta propia. Tabla elaborada por el SmartPLS V.3 
Los criterios de Validez y Confiabilidad del modelo corregido tienen valores según los parámetros recomendados, por lo que el modelo de este estudio es válido y confiable.

\subsubsection{Factores para la deserción universitaria}

El análisis de validez y confiabilidad del modelo indica que los factores Académico, Económico, Familiar, Motivacional, Salud y Social, influyen sobre la deserción en los estudiantes de la Universidad Mayor, Real y Pontificia de San Francisco Xavier de Chuquisaca.

Tabla 5. Variables de los factores que influyen sobre la deserción

\begin{tabular}{|c|c|c|c|}
\hline Factor & Cod & Ítem & Variable \\
\hline \multirow{2}{*}{ Académico } & $\mathrm{Ac03}$ & $\begin{array}{l}\text { Mis compañero/as de clase son más } \\
\text { inteligentes que yo }\end{array}$ & Autopercepción académica \\
\hline & Ac06 & $\begin{array}{l}\text { Las asignaturas que llevo me resultan muy } \\
\text { difíciles }\end{array}$ & Preparación académica \\
\hline \multirow[t]{2}{*}{ Económico } & Ec01 & $\begin{array}{l}\text { El dinero que tiene mi familia es suficiente } \\
\text { para que yo pueda estudiar }\end{array}$ & Soporte económico familiar \\
\hline & Ec05 & Necesito trabajar para poder estudiar & Necesidad de autofinanciamiento \\
\hline \multirow[t]{2}{*}{ Familiar } & $\mathrm{Fa07}$ & $\begin{array}{l}\text { Hay problemas en mi familia que perjudican } \\
\text { mis estudios }\end{array}$ & Conflictos familiares \\
\hline & $\mathrm{Fa} 09$ & Ser padre/madre me dificulta estudiar & Condición jefe de familia \\
\hline \multirow{2}{*}{ Motivacional } & Mo10 & Me siento acosado/a por mis docentes & Relación Docente-Estudiante \\
\hline & Mo11 & Me siento acosado/a por mis compañero/as & Relación Estudiante-Estudiante \\
\hline \multirow{2}{*}{ Salud } & $\mathrm{Sa} 01$ & Mi alimentación es nutritiva & Condición de alimentación \\
\hline & $\mathrm{Sa} 02$ & Tengo buen estado de salud & Estado general de salud \\
\hline \multirow[t]{2}{*}{ Social } & So01 & $\begin{array}{l}\text { La distancia de mi casa a mi facultad afecta } \\
\text { mi asistencia a clases }\end{array}$ & Acceso físico a la universidad \\
\hline & So07 & Me siento discriminado/a & Discriminación \\
\hline
\end{tabular}

Fuente: Encuesta sobre deserción universitaria 2017. Elaboración propia

Además, es posible identificar que algunos ítems de los factores tienen mayor influencia sobre la deserción. En la tabla 5 se muestran las variables que están relacionadas con los factores que influyen sobre la deserción en los estudiantes de la Universidad Mayor, Real y Pontificia de San Francisco Xavier de Chuquisaca. 


\section{Conclusión}

La investigación permite identificar los factores que influyen sobre la deserción universitaria en la Universidad de San Francisco Xavier de Chuquisaca, siendo estos factores relacionados con lo Académico, la Salud, lo Motivacional, lo Social, lo Económico y lo Familiar.

Existen variables que tienen mayor influencia sobre los factores, en ese sentido:

- En relación al factor Académico, el valor Beta ( $\beta$ ) es inferior al recomendado de 0.2 (Calvo-Porral, Martínez-Fernández, et al., 2013; Leyva, \& Olague, 2014; Ramirez-Correa \& Mariano, 2014), lo que significa que tiene un nivel de influencia bajo, menor al 10\% (0.092), significando que podría ser excluido del modelo estructural, pero desde un punto de vista del efecto socio-académico los investigadores consideran apropiado hacer un análisis sobre las variables que influyen sobre este factor; las variables de autopercepción académica y preparación académica son las que mayor influencia tienen sobre este factor. La autopercepción académica tiene relación con la comparación académica que realiza el estudiante con sus compañeros de aula, regularmente tiene relación con el acceso a información oportuna que puede ser resuelta con mejorar el acceso a bibliografía actualizada. En relación a la preparación académica, tiene que ver con la preparación previa y durante la estadía en la universidad, cursos de nivelación y de reforzamiento resultan ser medidas acertadas para afrontar este aspecto.

- El factor Económico tiene un factor beta con un nivel de influencia del $22.80 \%$ (0.228), por lo que tiene un nivel de influencia que debe ser considerado relevante para el modelo estructural que explica la deserción universitaria. Dos son las variables relevantes que influyen sobre el factor económico: soporte familiar y necesidad de autofinanciamiento. Soporte familiar, se refiere a la capacidad financiera que las familias tienen para mantener a los estudiantes durante su permanencia en la universidad, por los valores obtenidos se entiende que "el dinero de la familia no es suficiente para que pueda seguir estudiando"; la necesidad de autofinanciamiento hace referencia a la necesidad que los estudiantes tienen de buscar una fuente laboral para poder continuar sus estudios por la ausencia de apoyo familiar. Se recomienda realizar una investigación complementaria específica en relación a esta temática.

- El factor familiar tiene el más alto nivel de influencia sobre la deserción universitaria en el modelo estructural, con un valor Beta del 32.00\% (0.320); en el caso específico de la Universidad San Francisco Xavier de Chuquisaca, la influencia del entorno familiar es el de mayor influencia sobre la deserción universitaria. Dos son las variables que explican el factor familiar, conflictos 
familiares y condición jefe de familia. Los "conflictos familiares" se refiere a las relaciones en el contexto familiar del estudiante. La situación de "condición jefe de familia" está referida al hecho que los estudiantes son padres o madres de familia en condición de jefe de familia, esta situación es también externa a la Universidad, pero que tiene incidencia sobre la asistencia regular a las aulas universitarias. Al ser un factor con el más alto valor de influencia y por ser externa a la estructura se recomienda hacer un estudio complementario específico, que permita profundizar las caracterizaciones de ambas variables.

- Lo Motivacional es un factor con un nivel Beta de influencia del $12.10 \%$ (0.121), considerado bajo en el modelo estructural. Este factor es explicado por dos variables, la relación Docente-Estudiante y la relación EstudianteEstudiante, ambas tienen valores del factor Beta superiores al 80\% (D-E = 0.843 y E-E = 0.846). La relación Docente-Estudiante se refiere a la sensación de acoso por parte del Docente sobre el Estudiante, y en la relación Estudiante-Estudiante se refiere a la sensación de acoso por parte del Estudiante sobre el Estudiante. Es recomendable realizar un estudio complementario con esta temática específica.

- La Salud es un factor con nivel de influencia muy bajo sobre el modelo estructural que explica la deserción universitaria, el factor Beta es del 3.90\% (0.039).

- El factor Social, tiene un valor del factor Beta con valor igual a $11.60 \%$ (0.116), es considerado como bajo en el nivel de influencia sobre el constructo. Las variables que explican este factor están relacionados con un aspecto estructural de acceso físico a la universidad, y el relacionado con la percepción de discriminación de parte del estudiante. Se recomienda realizar un estudio complementario sobre esta temática.

Los factores, según el modelo, que tienen mayor influencia relativa sobre el constructo Deserción Estudiantil, son el factor Económico (22.80\%) y el factor Familiar (32.00\%).

\section{Referencias bibliográficas}

Balmori, E. E. R., de la Garza, M. T., \& Guzman, E. (2013). Diseño y validación de un instrumento para determinar las variables de deserción en los Institutos Tecnológicos. Pistas Educativas, 101, 291-302. 
Cabrera, L., Bethencourt, J. T., Alvarez, P., \& González, M. (2006). El problema del abandono de los estudios universitarios. RELIEVE REvista Electrónica de Investigación y Evaluación Educativa, 12(2), 171-203.

Calvo-Porral, C., Martínez-Fernández, V.-A., \& Juanatey-Boga, O. (2013). Análisis de dos modelos de ecuaciones estructurales alternativos para medir la intención de compra. Revista Investigación Operacional, 34(3), 230-243. Recuperado de http://bit.ly/2KIC9TA

Calvo-Porral, C., Martinez-Fernández, V. A., Juanatey-Boga, O., \& Lévy-Mangín, J. P. (2013). What matters to store Brand Equity? An approach to Spanish large retailing in a downturn context. Investigaciones Europeas de Direccion y Economia de La Empresa, 19(3), 136-146. https://doi.org/10.1016/j.iedee.2013.03.001

Celis, R., Flores, C. L., Reyes, M. C., \& Venegas, H. (2013). Factores de riesgo de deserción presentes en alumnos repitentes de las carreras de Enfermería y Kinesiología en una universidad chilena. Ciencia y Enfermería, 19(3), 63-71. https://doi. org/10.4067/S0717-95532013000300007

Centro Microdatos. (2008). Estudio sobre causas de la desercion universitaria. Centro Microdatos Departamento de Economía Universidad de Chile, 1-143.

CEUB, C. E. de la U. B. (2016). Boletín Estadístico. Boletín Estadístico Nº 5.

Da Re, L., \& Clerici, R. (2017). Abandono, rendimiento académico y tutoría: una investigación de la Universidad de Padua. Educatio Siglo XXI, 35(2 Julio), 139. https://doi. org/10.6018/j/298551

Escanés, G., Merlino, A., \& Ayllón, S. (2011). Variables que influyen en la deserción de estudiantes universitarios de primer año. Construcción de índices de riesgo de abandono. Revista Electrónica "Actualidades Investigativas En Educación," 11(2), 1-30. Recuperado de http://bit.ly/2rcich9

García de Fanelli, A., \& Adrogué de Deane, C. (2015). Abandono de los estudios universitarios: dimensión, factores asociados y desafíos para la política pública. Revista Fuentes, 16, 85-106. https://doi.org/10.12795/revistafuentes.2015.116.04

González-Ramírez, T., \& Pedraza-Navarro, I. (2017). Variables sociofamiliares asociadas al abandono de los estudios universitarios. Educatio Siglo XXI, 35(2 Julio), 365. https://doi.org/10.6018///298651

Ledesma, R. (2008). Introduccción al Bootstrap. Desarrollo de un ejemplo acompañado de software de aplicación Rubén. Tutorials in Quantitative Methods for Psychology, 4(2), 51-60. https://doi.org/10.20982/tamp.04.2.p051

Leyva, O., \& Olague, J. T. (2014). Modelo de Ecuaciones Estructurales por el Método de Mínimos Cuadrados Parciales (Partial Least Squares-PLS). TIRANT HUMANIDADES MÉXICO - Métodos y Técnicas Cualitativas y Cuantitativas Aplicables a la Investigación en Ciencias Sociales.

Martínez, J. A., \& Martínez, L. (2009). Los test estadísticos y la evaluación de escalas; el caso de la validez discriminante. Investigaciones Europeas de Direccion y Economia de La Empresa, 15(2), 15-24. https://doi.org/10.1016/S1135-2523(12)60086-0 
Medellín, E. W. (2010). Contrast Between Two Self-Determination Motivational Models in Order To Predict University Students' Dropout. Acta Colombiana de Psicología, 13(2), 57-68. Recuperado de http://bit.ly/2s4V84s

Mendez-Gimenez, A., Fernández-Río, J., \& Cecchini-Estrada, J.-A. (2014). Validación de la versión en español del Cuestionario de Metas de Amistad en Educación Física. Universitas Psychologica, 13(1), 227-237. https://doi.org/10.11144/Javeriana. UPSY13-1.vvec

Miranda, A. (2003). El Método de Remuestreo y su Aplicación en la Investigación Biomédica.

Morles, V. (2002). Sobre la metodología como ciencia y el método científico: un espacio polémico. Revista de Pedagogía, 23(66), 16.

Morone, G. (2015). Métodos y técnicas de la investigación científica.

Piratoba H., B. N., \& Barbosa Ch., O. O. (2013). Factores de deserción de los estudiantes en la Facultad de Enfermería de la Universidad de ciencias aplicadas y ambientales UDCA, durante el periodo 2009 a 2011. Revista U.D.C.A Actualidad \& Divulgación, 16(2), 553-562.

Ramirez-Correa, P. E., \& Mariano, A. M. (2014). Propuesta metodológica para aplicar modelos de ecuaciones estructurales con PLS: El caso del uso de bases de datos científicas en estudiantes universitarios. Revista ADMpg Gestão Estratégica, 7(2), 133-139. Recuperado de http://bit.ly/37q8BDY

Ramos, N. (2011). Deserción universitaria. Monografias.Com, 21.

Robles, F. (2018). Tipos de Investigación. Recuperado de http://bit.ly/35id9KI

Rodriguez, G., \& Weise, C. (2006). Educacion Superior Universitaria en Bolivia - Estudio Nacional.

Ruiz, R. (2007). El Método Científico y su Etapas. México DF.

Sabino, C. (1992). El proceso de investigacion. Bueno Aires, Argentina.

Sánchez, G., Navarro, W., \& García, A. D. (2009). Factores de deserción estudiantil en la Universidad Surcolombiana. Revista Paideia Surcolombiana, 14, 97-103.

Siles, E. W., \& Cortez, S. G. (2003). Deserción estudiantil en la Universidad Autónoma Gabriel René Moreno.

Solano, J. A., Pérez, M. J., \& Uzcátegui, C. de los Á. (2017). Metodologías de Medición de la Competitividad. In I/ Congreso Internacional Virtual sobre desafíos de las empresas del siglo XXI (pp. 123-130).

Tinto, V. (1993). Definir la deserción: una cuestión de perspectiva.

UMRPSFXCH/Dirección de Servicios Académicos, D. (2016). Universidad Mayor, Real y Pontificia de San Francisco Xavier de Chuquisaca. Estadísticas de rendimiento academico del curso normal por carrera. Gestiones 2014 - 2016. 
Análisis de la deserción estudiantil en una universidad pública de Bolivia.

UMRPSFXCH/Dirección de Tecnologías de Información y Comunicación. (2017). Universidad Mayor, Real y Pontificia de San Francisco Xavier de Chuquisaca. Estadísticas sobre evolución de la matrícula regular. Gestiones 2013 - 2017. DTIC.

Viale, H. E. (2014). Una aproximación teórica a la deserción estudiantil universitaria. Revista Digital de Investigación En Docencia Universitaria (RIDU), 8(1), 59-75. Recuperado de http://bit.ly/2rgnB76

Vries, W. de, León, P., Romero, J. F., \& Hernandez, I. (2011). ¿Desertores o decepcionados? Distintas causas para abandonar los estudios universitarios. Revista De La Educación Superior, XL(4)(160), 29-49. https://doi.org/0185-2760 


\section{Anexos}

\begin{tabular}{|c|c|c|c|c|c|c|c|}
\hline \multicolumn{8}{|c|}{ Anexo 1. Encuesta final } \\
\hline Factor & Cod & Afirmación & 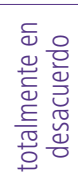 & 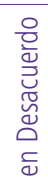 & 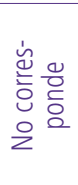 & $\begin{array}{l}\text { 응 } \\
\frac{0}{3} \\
\frac{4}{4} \\
\frac{0}{0}\end{array}$ & 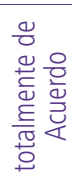 \\
\hline \multirow{5}{*}{ 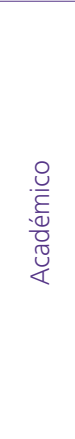 } & $\mathrm{AcO} 2$ & $\begin{array}{l}\text { Comprendo bien el conocimiento que } \\
\text { imparten mis docentes }\end{array}$ & & & & & \\
\hline & $\mathrm{Ac03}$ & $\begin{array}{l}\text { Mis compañero/as de clase son más } \\
\text { inteligentes que yo }\end{array}$ & & & & & \\
\hline & Ac06 & $\begin{array}{l}\text { Las asignaturas que llevo me resultan muy } \\
\text { difíciles }\end{array}$ & & & & & \\
\hline & Ac10 & $\begin{array}{l}\text { Necesito ayuda para estudiar algunas } \\
\text { materias }\end{array}$ & & & & & \\
\hline & Ac13 & $\begin{array}{l}\text { Tengo dificultad para resolver ejercicios y/o } \\
\text { prácticas }\end{array}$ & & & & & \\
\hline \multirow{3}{*}{ 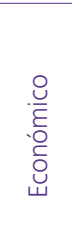 } & Ec01 & $\begin{array}{l}\text { El dinero que tiene mi familia es suficiente } \\
\text { para que yo pueda estudiar }\end{array}$ & & & & & \\
\hline & Ec03 & $\begin{array}{l}\text { Ocupo mucho tiempo en otras actividades } \\
\text { en vez de estudiar }\end{array}$ & & & & & \\
\hline & Ec05 & Necesito trabajar para poder estudiar & & & & & \\
\hline \multirow{5}{*}{ 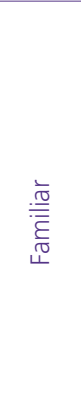 } & $\mathrm{FaO2}$ & $\begin{array}{l}\text { En mi familia casi todos tienen la misma } \\
\text { profesión que estoy estudiando }\end{array}$ & & & & & \\
\hline & $\mathrm{FaO3}$ & Extraño vivir con mi familia & & & & & \\
\hline & $\mathrm{FaO}$ & $\begin{array}{l}\text { Hay problemas en mi familia que perjudican } \\
\text { mis estudios }\end{array}$ & & & & & \\
\hline & $\mathrm{Fa08}$ & Recibo maltrato de mi familia & & & & & \\
\hline & $\mathrm{Fa09}$ & Ser padre/madre me dificulta estudiar & & & & & \\
\hline \multirow{4}{*}{ 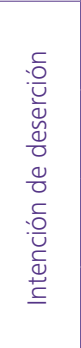 } & $\ln 02$ & $\begin{array}{l}\text { Mi situación económica no permite que } \\
\text { siga estudiando }\end{array}$ & & & & & \\
\hline & $\ln 03$ & Por razones de salud debo dejar de estudiar & & & & & \\
\hline & $\ln 04$ & $\begin{array}{l}\text { Dejaré mis estudios porque es una carrera } \\
\text { muy difícil }\end{array}$ & & & & & \\
\hline & $\ln 05$ & $\begin{array}{l}\text { No puedo seguir estudiando, porque debo } \\
\text { mantener a mi familia }\end{array}$ & & & & & \\
\hline
\end{tabular}




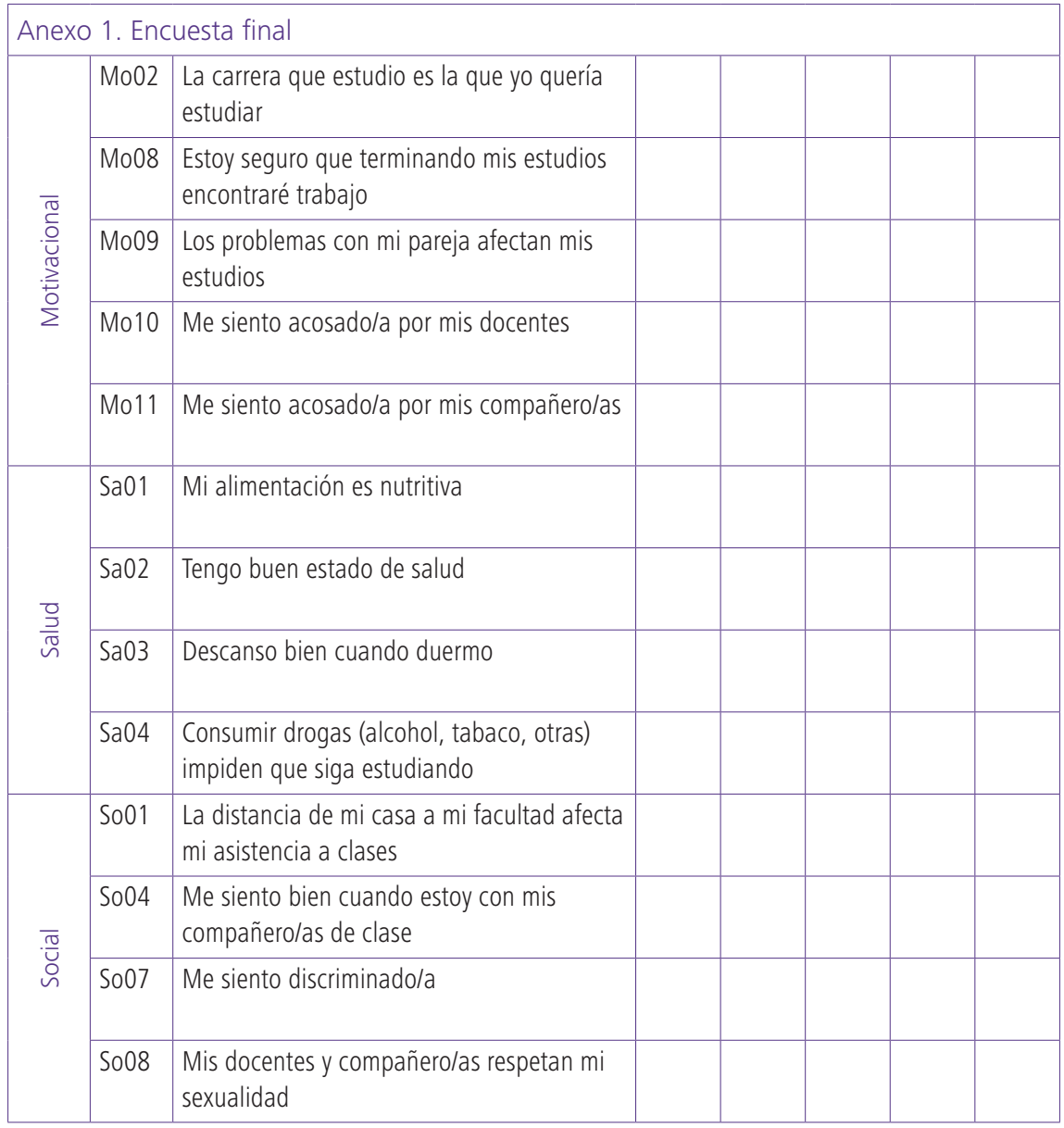

